\title{
Chromatographie Sur Couche Mince Et Activité Antiradicalaire D'extraits De Pupalia Lappacea (L.) Juss. Amaranthaceae
}

\author{
Mamadou Aïssa Jazy \\ Laboratoire de Botanique- Pharmacognosie, Faculté des Sciences de la Santé, \\ Université Abdou Moumouni, Niamey-Niger

\section{Mahamane Haïdara, Rokia Sanogo} \\ DER des Sciences Pharmaceutiques, Faculté de Pharmacie, Université des \\ Sciences, Techniques et Technologies de Bamako-Mali. Département de \\ Médecine Traditionnelle, Institut National de Recherche en Santé Publique, \\ Ministère de la Santé Publique, Bamako-Mali
}

Doi: 10.19044/esj.2018.v14n3p140 URL:http://dx.doi.org/10.19044/esj.2018.v14n3p140

\begin{abstract}
Introduction: $P$. lappacea is a plant used in traditional medicine, in the regions of Niamey and Tillaberi, as anti-hemorrhoids. It contains tannins, sterols, triterpenes, saponosides, mucilages, anthracene derivatives, oses and holosides for which it is necessary to appreciate the activities relating to the medicinal virtues. Objective: To contribute to the phytochemical and pharmacological study of Pupalia lappacea, through the semi-quantitative estimation of phytocomposés and the research of the antiradical power of its various extracts. Methodology: Phytochemical screening, semiquantitative estimation and a study of the antioxidant activity of phytocomposites were carried out by thin layer chromatography (TLC) from extracts obtained with increasing polarity solvents. Results: All P. lappacea extracts contain a large number of bioactive chemical compounds. Substances with antihaemorrhoidal activity (phenolic compounds, tannins) are found mainly in the aqueous extracts (digested, decoct exhausted) and the methanolic extract. The strongest antiradical activity observed with the digest and the spent decoction after migration in the solvent system. Ethyl acetate - Methyl ethyl ketone - Formic acid - Water (5-3-1-1) would be linked to phenolic compounds. A less severe free radical activity, probably due to terpenic substances, was observed with the BAW solvent system (60-15-25). The extract corresponding to the traditional (decocted) use of $P$. lappacea also has low antioxidant activity. Conclusion: The use of $P$. lappacea as antihemorrhoid is justified. However, the digest and the spent decoction would have greater anti-hemorrhoidal properties than those of the decoction obtained
\end{abstract}


by the traditional method. An assay of the phenolic compounds, tannins, terpenic compounds, in the infused, the decoction, the digested and the exhausted decoction would make it possible to choose the best method of extraction and to improve the anti-hemorrhoidal effect of the $P$. lappacea based medicinal preparations.

Keywords: Pupalia lappacea, CCM, phenolic compounds, terpenes, antiradical activity

\section{Résumé}

Introduction : Pupalia lappacea est une plante utilisée en médecine traditionnelle, dans les régions de Niamey et Tillabéri, comme antihémorroïdaire. Elle renferme des tanins, stérols, triterpènes, saponosides, mucilages, dérivés anthracéniques, oses et holosides pour lesquels il faut apprécier les activités relatives aux vertus médicinales. Objectif : Contribuer à l'étude phytochimique et pharmacologique de $P$. lappacea, à travers l'estimation semi-quantitative des phytocomposés et la recherche du pouvoir antiradicalaire de ses divers extraits. Méthodologie : Nous avons effectué par chromatographie sur couche mince (CCM) un criblage phytochimique, une estimation semi-quantitative ainsi qu'une étude de l'activité antioxydante des phytocomposés, à partir d'extraits obtenus avec des solvants à polarité croissante. Résultats : Tous les extraits de $P$. lappacea renferment un grand nombre de composés chimiques bioactifs. Les substances à activité antihémorroïdaire (composés phénoliques, tanins) se retrouvent surtout dans les extraits aqueux (digesté, décocté épuisé) et l'extrait méthanolique. La plus forte activité antiradicalaire observée avec le digesté et le décocté épuisé après migration dans le système de solvants Acétate d'éthyle - Méthyléthylcétone Acide formique - Eau (5-3-1-1) serait liée aux composés phénoliques. Une activité antiradicalaire moins importante, probablement due aux substances terpéniques, a été constatée avec le système de solvants BAW (60-15-25). L'extrait correspondant à l'emploi traditionnel (décocté) de $P$. lappacea présente également une faible activité antioxydante. Conclusion : L'usage de $P$. lappacea comme antihémorroïdaire est justifié. Cependant, le digesté et le décocté épuisé possèderaient des propriétés antihémorroïdaires plus importantes que celles du décocté obtenu par la méthode traditionnelle. Un dosage des composés phénoliques, tanins, composés terpéniques, dans l'infusé, le décocté, le digesté et le décocté épuisé permettrait de choisir la meilleure méthode d'extraction et d'améliorer l'effet antihémorroïdaire des préparations médicinales à base de $P$. lappacea.

Mots-clés : Pupalia lappacea, CCM, composés phénoliques, terpènes, activité antiradicalaire 


\section{Introduction}

Une étude antérieure de $P$. lappacea, plante utilisée en médecine traditionnelle comme anti-hémorrö̈daire, a montré qu'elle renferme des métabolites secondaires bioactifs qui, par leurs propriétés, peuvent soigner les symptômes des hémorroïdes (Mamadou et al, 2017 a et b). Ce sont des tanins, stérols et triterpènes, saponosides, mucilages, dérivés anthracéniques, oses et holosides.

Dans la même lancée, nous avons jugé utile d'apprécier la richesse en substances chimiques de cette plante et d'étudier une activité biologique pouvant renseigner sur son efficacité dans le traitement des hémorroïdes. Parmi les activités biologiques qui peuvent donner un effet antihémorroïdaire figure l'activité antioxydante.

La présente étude est une contribution à la valorisation des plantes médicinales antihémorroïdaires du Niger. Elle vise à évaluer la richesse de $P$. lappacea en composés chimiques et à rechercher une activité antioxydante qui pourraient justifier l'utilisation traditionnelle de cette plante.

\section{Méthodologie}

Après extraction par divers solvants, nous avons effectué une analyse phytochimique semi-quantitative par chromatographie sur couche mince (CCM).

Elle a permis de faire un criblage chimique des composés contenus dans les extraits, de connaître leur abondance et d'évaluer le pouvoir antiradicalaire.

\section{Extractions}

Les extractions des substances chimiques ont été faites avec des solvants organiques à polarité croissante. Nous avons réalisé, pour chaque échantillon, une macération avec successivement: éther de pétrole (E1), dichlorométhane (E2), acétate d'éthyle (E3), méthanol (E4). Le marc est repris avec de l'eau $(100 \mathrm{ml})$ et chauffé à $50{ }^{\circ} \mathrm{C}$ pendant $30 \mathrm{mn}$; l'infusé $(\mathrm{E} 5)$ est recueilli. Enfin le dernier marc qui a reçu à son tour de l'eau $(100 \mathrm{ml})$ est chauffé à $100{ }^{\circ} \mathrm{C}$ pendant $30 \mathrm{mn}$; le décocté (E6) est recueilli après refroidissement.

\section{Chromatographie sur couche mince \\ Principe de la CCM}

Il repose sur la séparation des substances chimiques par migration et adsorption sur un support ou phase stationnaire polaire, dans une phase mobile ou éluant, en fonction de leur nature, du pouvoir éluant de la phase mobile, du pouvoir adsorbant du support. 


\section{Technique}

La phase stationnaire est une couche de gel de silice $60 \mathrm{~F} 254$ fixée sur une plaque en aluminium 20x20 cm. L'éluant ou système de migration est un mélange de solvants. Nous avons utilisé 3 systèmes de solvants pour les extraits organiques de nos échantillons où se retrouvent les constituants apolaires à peu polaires : le système éther de pétrole - acétate d'éthyle (2-1), le système éther de pétrole - acétate d'éthyle (1-1), le système acétate d'éthyle - méthyléthyl cétone - acide formique - eau (5-3-1-1). Les extraits aqueux qui renferment les constituants peu polaires à polaires ont été chromatographiés avec le système acétate d'éthyle - méthyl éthyl cétone - acide formique - eau (5-3-1-1), le système butanol - acide acétique - eau (40-10-50) et le système butanol - acide acétique - eau (60 - 15 - 25).

\section{Screening chimique}

Nous avons recherché principalement les phytocomposés à propriétés antihémorroïdaires. La révélation a été faite avec :

- le réactif de Godin qui permet de détecter la présence de diverses substances parmi lesquelles les stérols, terpènes, flavonoïdes, coumarines, saponosides; -l'anisaldéhyde, qui met en évidence également plusieurs composés (saponosides, triterpènes...) ;

- le chlorure chlorure de fer à $2 \%$ pour les composés phénoliques, les tanins ; -le chlorure d'aluminium $\left(\mathrm{AlCl}_{3}\right)$ à $1 \%$ concernant les flavonoïdes, les lactones sesquiterpéniques (Sofowora, 1993 ; Harborne, 1998 ; OUA, 1988).

\section{Détection du pouvoir antiradicalaire}

Nous avons évalué le pouvoir antioxydant des extraits à partir des plaques de CCM selon la méthode de Takao (1994). L'antioxydant de référence utilisé est le 1,1- diphényl-2-picrylhydrazyle ou DPPH. C'est un radical libre stable de couleur violacée qui s'éclaircit en présence d'un composé antioxydant. L'efficacité antioxydante d'un composé est mesurée par sa capacité à décolorer le DPPH qui devient jaune; elle est proportionnelle à l'intensité de la décoloration.

Les profils chromatographiques du criblage chimique ont été comparés à ceux de l'activité antioxydante.

\section{Résultats}

\section{Criblage des phytocomposés}

Les tableaux I à VIII présentent les distances parcourues par les différents composés, les colorations observées, les nombres et groupes de composés identifiés.

- Système de solvants Ether de pétrole/Acétate d'éthyle (1-1) 
Les lactones sesquiterpéniques révélées par le chlorure d'aluminium (tableau I) sont surtout dans l'extrait de dichlorométhane (6 composés).

Après révélation par l'anisaldéhyde, les saponosides essentiellement ont été mis en évidence. Les extraits d'acétate d'éthyle, de dichlorométhane et de méthanol renferment plus de composés révélés par l'anisaldéhyde que l'extrait d'éther de pétrole avec respectivement 5, 5, 6, et 4 composés (tableau II et figure 1). Le nombre total de composés est moins élevé dans l'extrait d'éther de pétrole ( 7 composés) alors qu'il est de 11,9 et 11 composés dans les extraits de dichlorométhane, acétate d'éthyle et méthanol respectivement.

Tableau I : Observation du chromatogramme avant et après révélation par le chlorure d'aluminium (AlCl3)

\begin{tabular}{|c|c|c|c|c|c|c|}
\hline $\mathrm{Rf}$ & $254 \mathrm{~nm}$ & $\begin{array}{c}366 \mathrm{~nm} \\
\text { fluorescence }\end{array}$ & $\mathrm{AlCl}_{3}$ & $\begin{array}{c}\text { Nombre } \\
\text { de } \\
\text { composés } \\
\text { identifiés }\end{array}$ & $\begin{array}{c}\text { Groupes } \\
\text { de } \\
\text { composés } \\
\text { identifiés }\end{array}$ & $\begin{array}{l}\text { Nombre } \\
\text { total } \\
\text { de } \\
\text { composés }\end{array}$ \\
\hline \multicolumn{7}{|c|}{ E1 (Ether de pétrole) } \\
\hline 0,65 & & & Bleu & \multirow{4}{*}{4} & \multirow{25}{*}{$\begin{array}{c}\text { Lactones } \\
\text { sesquiterpéniques }\end{array}$} & \multirow{4}{*}{4} \\
\hline 0,78 & & Bleu & Rouge & & & \\
\hline 0,88 & Visible & Rouge & Rouge & & & \\
\hline 0,94 & Visible & Rouge & Bleu & & & \\
\hline \multicolumn{4}{|c|}{ E2 (Dichlorométhane) } & \multirow{9}{*}{6} & & \multirow{9}{*}{7} \\
\hline 0,08 & Visible & Rouge & Rouge & & & \\
\hline 0,45 & Visible & & & & & \\
\hline 0,56 & Visible & Rouge & Rouge & & & \\
\hline 0,69 & & & Bleu & & & \\
\hline 0,78 & & Rouge & Bleu & & & \\
\hline \multirow[t]{2}{*}{0,88} & Visible & Rouge & & & & \\
\hline & & & Rouge & & & \\
\hline 0,95 & Visible & Rouge & Bleu & & & \\
\hline \multicolumn{4}{|c|}{ E3 (Acétate d'éthyle) } & \multirow{6}{*}{5} & & \multirow{6}{*}{5} \\
\hline 0,07 & Visible & Rouge & Rouge & & & \\
\hline 0,44 & Visible & Rouge & Rouge & & & \\
\hline 0,59 & Visible & Rouge & Rouge & & & \\
\hline 0,88 & Visible & Rouge & Rouge & & & \\
\hline 0,96 & Visible & Rouge & Bleu & & & \\
\hline \multicolumn{4}{|c|}{ E4 (Méthanol) } & \multirow{6}{*}{5} & & \multirow{6}{*}{5} \\
\hline 0,31 & & Rouge & Bleu & & & \\
\hline 0,48 & Visible & Rouge & Rouge & & & \\
\hline 0,63 & Visible & Rouge & Rouge & & & \\
\hline 0,78 & & Rouge & Bleu & & & \\
\hline 0,93 & Visible & Rouge & Rouge & & & \\
\hline
\end{tabular}




\begin{tabular}{|c|c|c|c|c|c|c|}
\hline $\mathrm{Rf}$ & $254 \mathrm{~nm}$ & $\begin{array}{c}366 \mathrm{~nm} \\
\text { fluorescence }\end{array}$ & Anisaldéhyde & $\begin{array}{c}\text { Nombre } \\
\text { de } \\
\text { composés } \\
\text { identifiés }\end{array}$ & $\begin{array}{c}\text { Groupes } \\
\text { de } \\
\text { composés } \\
\text { identifiés }\end{array}$ & $\begin{array}{l}\text { Nombre } \\
\text { total de } \\
\text { composés }\end{array}$ \\
\hline \multicolumn{7}{|c|}{ E1 (Ether de pétrole) } \\
\hline 0,69 & & & Noir & \multirow[t]{7}{*}{4} & \multirow{7}{*}{$\begin{array}{l}\text { Saponosides } \\
\text { Triterpènes }\end{array}$} & \multirow[t]{7}{*}{7} \\
\hline 0,78 & & Bleu & & & & \\
\hline 0,83 & & & Noir & & & \\
\hline 0,88 & Visible & Rouge & & & & \\
\hline 0,91 & & & Noir & & & \\
\hline 0,94 & Visible & Rouge & & & & \\
\hline 0,96 & & & Noir & & & \\
\hline \multicolumn{7}{|c|}{ E2 (DCM) } \\
\hline 0,08 & Visible & Rouge & & \multirow{11}{*}{5} & \multirow{11}{*}{ Saponosides } & \multirow{11}{*}{11} \\
\hline 0,1 & & & Noir & & & \\
\hline 0,45 & Visible & & & & & \\
\hline 0,56 & Visible & Rouge & & & & \\
\hline 0,69 & & & Noir & & & \\
\hline 0,78 & & Rouge & & & & \\
\hline 0,81 & & & Noir & & & \\
\hline 0,88 & Visible & Rouge & & & & \\
\hline 0,91 & & & Noir & & & \\
\hline 0,95 & Visible & Rouge & & & & \\
\hline 0,96 & & & Noir & & & \\
\hline \multicolumn{7}{|c|}{ E3 (Acétate d'éthyle) } \\
\hline 0,07 & visible & rouge & & \multirow{9}{*}{5} & \multirow[t]{9}{*}{ Saponosides } & \multirow{9}{*}{9} \\
\hline 0,09 & & & Noir & & & \\
\hline 0,41 & & & Noir & & & \\
\hline 0,44 & visible & rouge & & & & \\
\hline 0,59 & visible & rouge & & & & \\
\hline 0,69 & & & Noir & & & \\
\hline 0,88 & visible & rouge & Noir & & & \\
\hline 0,96 & visible & rouge & & & & \\
\hline 0,98 & & & Noir & & & \\
\hline \multicolumn{6}{|c|}{ E4 (Méthanol) } & \\
\hline 0,25 & & & Noir & \multirow{11}{*}{6} & \multirow[t]{11}{*}{ Saponosides } & \multirow{11}{*}{11} \\
\hline 0,31 & & rouge & & & & \\
\hline 0,44 & & & Noir & & & \\
\hline 0,48 & visible & rouge & & & & \\
\hline 0,58 & & & Vert & & & \\
\hline 0,63 & visible & rouge & & & & \\
\hline 0,69 & & & Noir & & & \\
\hline 0,78 & & rouge & & & & \\
\hline 0,89 & & & Vert & & & \\
\hline 0,93 & visible & rouge & & & & \\
\hline 0,98 & & & Noir & & & \\
\hline
\end{tabular}

Tableau II: Observation du chromatogramme avant et après révélation par l'anisaldéhyde 


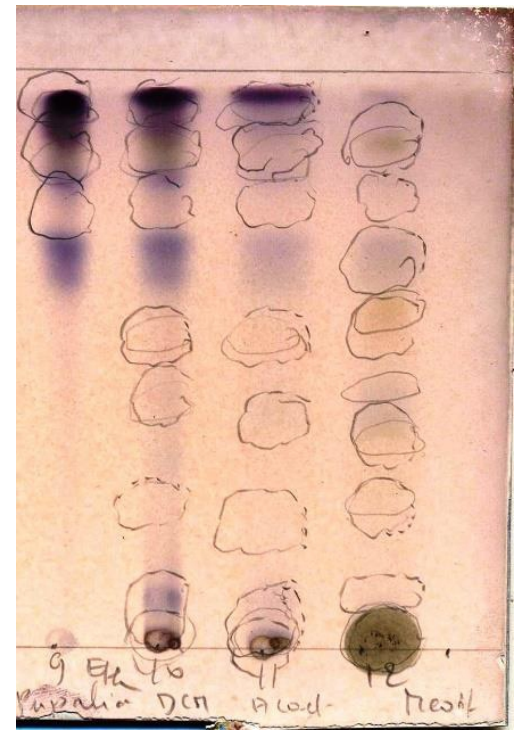

Figure 1 : Saponosides et terpènes révélés dans le visible par l'anisaldéhyde - $\quad$ Phase mobile : Ether de pétrole - Acétate d'éthyle (2-1).

Les saponosides, stérols et Terpènes mis en évidence par le réactif de Godin (Figure 2 et tableau III) se retrouvent majoritairement dans les extraits d'éther de pétrole et de dichlorométhane (4 composés chacun); dans l'ensemble, les phytocomposés sont plus nombreux dans les extraits de dichlorométhane, de méthanol (8 composés chacun), et d'acétate d'éthyle (7 composés).

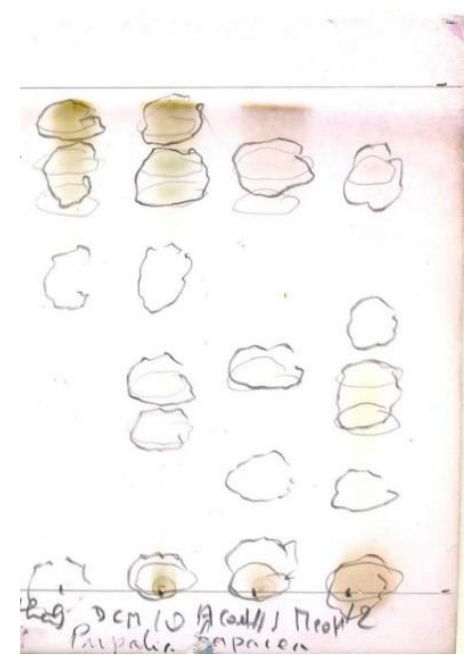

Figure 2 : Saponosides, Stérols et Terpènes révélés par le réactif de Godin dans le visible 
Tableau III: Observation du chromatogramme avant et après révélation par le réactif de Godin

\begin{tabular}{|c|c|c|c|c|c|c|}
\hline $\mathrm{Rf}$ & $\begin{array}{l}254 \\
\mathrm{~nm}\end{array}$ & $\begin{array}{c}366 \mathrm{~nm} \\
\text { fluorescence }\end{array}$ & $\begin{array}{l}\text { Réactif de } \\
\text { Godin }\end{array}$ & $\begin{array}{l}\text { Nombre } \\
\text { de } \\
\text { composés } \\
\text { identifiés }\end{array}$ & $\begin{array}{l}\text { Groupes } \\
\text { de } \\
\text { composés } \\
\text { identifiés }\end{array}$ & $\begin{array}{l}\text { Nombre } \\
\text { total de } \\
\text { composés }\end{array}$ \\
\hline \multicolumn{7}{|c|}{ E1 (Ether de pétrole) } \\
\hline 0,63 & & Rouge & Bleu-violacé & \multirow{5}{*}{4} & \multirow{5}{*}{$\begin{array}{c}\text { Saponosides } \\
\text { Stérols et } \\
\text { Terpènes }\end{array}$} & \multirow{5}{*}{5} \\
\hline 0,79 & Visible & & Bleu-violacé & & & \\
\hline 0,84 & & Rouge & Bleu-violacé & & & \\
\hline 0,88 & Visible & & & & & \\
\hline 0,95 & Visible & Rouge & Vert & & & \\
\hline \multicolumn{7}{|c|}{ E2 (DCM) } \\
\hline 0,33 & Visible & Rouge & & \multirow{8}{*}{4} & \multirow{8}{*}{$\begin{array}{c}\text { Saponosides } \\
\text { Stérols et } \\
\text { Terpènes }\end{array}$} & \multirow{8}{*}{8} \\
\hline 0,43 & Visible & Rouge & & & & \\
\hline 0,63 & & Rouge & Vert & & & \\
\hline 0,69 & & & Vert & & & \\
\hline 0,8 & Visible & & & & & \\
\hline 0,81 & & Rouge & Bleu-violacé & & & \\
\hline 0,85 & Visible & & & & & \\
\hline 0,94 & Visible & Rouge & Vert & & & \\
\hline \multicolumn{7}{|c|}{ E3 (Acétate d'éthyle) } \\
\hline 0,06 & & Rouge & & \multirow{7}{*}{3} & \multirow{7}{*}{$\begin{array}{c}\text { Saponosides } \\
\text { Stérols et } \\
\text { Terpènes }\end{array}$} & \multirow{7}{*}{7} \\
\hline 0,23 & & Rouge & & & & \\
\hline 0,44 & Visible & Rouge & & & & \\
\hline 0,69 & & & Bleu-violacé & & & \\
\hline 0,79 & Visible & & & & & \\
\hline 0,85 & Visible & Rouge & Bleu-violacé & & & \\
\hline 0,95 & & & Vert & & & \\
\hline \multicolumn{7}{|c|}{ E4 (Méthanol) } \\
\hline 0,2 & & Rouge & & \multirow{8}{*}{2} & \multirow[t]{8}{*}{ Saponosides } & \multirow{8}{*}{8} \\
\hline 0,34 & Visible & & & & & \\
\hline 0,38 & & Rouge & & & & \\
\hline 0,44 & Visible & & & & & \\
\hline 0,54 & & Rouge & & & & \\
\hline 0,66 & & & Bleu-violacé & & & \\
\hline 0,84 & Visible & Rouge & & & & \\
\hline 0,91 & & & Bleu-violacé & & & \\
\hline
\end{tabular}

- Phase mobile : Acétate d'éthyle - Méthyléthylcétone - Acide formique Eau (5-3-1-1)

Les tanins et autres composés phénoliques révélés par le chlorure de fer (tableau IV et figures 3 et $6 \mathrm{~A}$ ) sont surtout présents dans les extraits aqueux (digesté, décocté épuisé) avec 3 composés chacun. Au total, il y a 9 composés pour le décocté épuisé, 7 pour le digesté et 7 dans l'extrait méthanolique. 
Tableau IV : Observation du chromatogramme avant et après révélation par le Chlorure de fer $(\mathrm{FeCl} 3)$

\begin{tabular}{|c|c|c|c|c|c|c|}
\hline $\mathrm{Rf}$ & $254 \mathrm{~nm}$ & $\begin{array}{c}366 \mathrm{~nm} \\
\text { fluorescence }\end{array}$ & $\mathrm{FeCl}_{3}$ & $\begin{array}{c}\text { Nombre } \\
\text { de } \\
\text { composés } \\
\text { identifiés }\end{array}$ & \begin{tabular}{l} 
Groupes \\
\multicolumn{1}{c}{ de } \\
composés \\
identifiés
\end{tabular} & $\begin{array}{c}\text { Nombre } \\
\text { total de } \\
\text { composés }\end{array}$ \\
\hline \multicolumn{7}{|c|}{ E1 (Ether de pétrole) } \\
\hline 0,88 & Visible & Rouge & Vert & 1 & $\begin{array}{c}\text { Tanins, } \\
\text { Composés } \\
\text { phénoliques }\end{array}$ & 1 \\
\hline \multicolumn{7}{|c|}{ E2 (DCM) } \\
\hline 088 & Visible & Rouge & Vert & 1 & $\begin{array}{l}\text { Tanins, } \\
\text { Composés } \\
\text { phénoliques }\end{array}$ & 1 \\
\hline \multicolumn{7}{|c|}{ E3 (Acétate d'éthyle) } \\
\hline 0,66 & Visible & & & \multirow[b]{2}{*}{1} & \multirow{2}{*}{$\begin{array}{c}\text { Tanins, } \\
\text { Composés } \\
\text { phénoliques } \\
\end{array}$} & \multirow{3}{*}{7} \\
\hline 088 & Visible & Rouge & Vert & & & \\
\hline \multicolumn{7}{|c|}{ E4 (Méthanol) } \\
\hline 0,13 & Visible & & & \multirow{7}{*}{1} & \multirow{7}{*}{$\begin{array}{c}\text { Tanins, } \\
\text { Composés } \\
\text { phénoliques }\end{array}$} & \multirow{7}{*}{7} \\
\hline 029 & Visible & & & & & \\
\hline 0,38 & & Bleu & & & & \\
\hline 0,41 & Visible & & & & & \\
\hline 0,56 & & Bleu & & & & \\
\hline 0,69 & Visible & Bleu & & & & \\
\hline 0,88 & Visible & Rouge & Vert & & & \\
\hline \multicolumn{6}{|c|}{ E5 (Digesté $50^{\circ} \mathrm{C}$ ) } & \\
\hline 0,09 & & Violet & & \multirow{7}{*}{3} & \multirow{7}{*}{$\begin{array}{l}\text { Composés } \\
\text { phénoliques }\end{array}$} & \multirow{7}{*}{7} \\
\hline 0,21 & & Violet & Bleu & & & \\
\hline 0,33 & Visible & & & & & \\
\hline 0,46 & & & Bleu & & & \\
\hline 0,59 & Visible & Vert & & & & \\
\hline 0,75 & Visible & Rouge & Bleu & & & \\
\hline 0,94 & Visible & Rouge & & & & \\
\hline \multicolumn{7}{|c|}{ E6 (Décocté épuisé) } \\
\hline 0,09 & & Violet & & \multirow{9}{*}{3} & \multirow{9}{*}{$\begin{array}{l}\text { Composés } \\
\text { phénoliques }\end{array}$} & \multirow{9}{*}{9} \\
\hline 0,18 & & & Bleu & & & \\
\hline 0,23 & Visible & & & & & \\
\hline 0,46 & & & Bleu & & & \\
\hline 0,58 & Visible & Vert & & & & \\
\hline 0,75 & Visible & Rouge & & & & \\
\hline 0,78 & & & Bleu & & & \\
\hline 0,88 & Visible & Rouge & & & & \\
\hline 0,94 & & Rouge & & & & \\
\hline
\end{tabular}




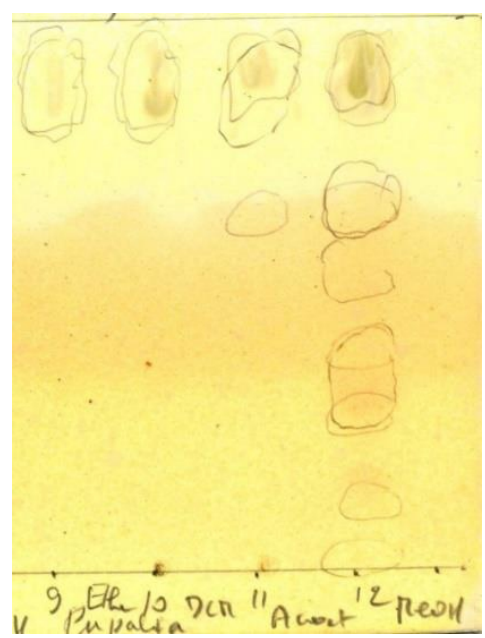

Figure 3 : Tanins et autres Composés phénoliques révélés dans le visible par le chlorure de fer

- Eluant : Butanol -Acide acétique - Eau (60-15-25)

Le digesté contient plus de composés terpéniques après révélation par le réactif de Godin (3 composés) que le décocté épuisé. Cependant, ils renferment tous beaucoup de substances avec 8 composés chacun (tableau $\mathrm{V}$ et figure $5 \mathrm{~A}$ ).

Tableau V : Observation du chromatogramme avant et après révélation par le réactif de Godin

\begin{tabular}{|c|c|c|c|c|c|}
\hline $\mathrm{Rf}$ & $254 \mathrm{~nm}$ & Godin & $\begin{array}{c}\text { Nombre } \\
\text { de composés } \\
\text { identifiés }\end{array}$ & $\begin{array}{c}\text { Groupes } \\
\text { de composés } \\
\text { identifiés }\end{array}$ & $\begin{array}{l}\text { Nombre total } \\
\text { de composés }\end{array}$ \\
\hline \multicolumn{5}{|c|}{ Digesté $50^{\circ} \mathrm{C}$} & \multirow{9}{*}{8} \\
\hline 0,05 & Visible & & \multirow{8}{*}{3} & \multirow[t]{8}{*}{ Terpènes } & \\
\hline 0,08 & & & & & \\
\hline 0,19 & Visible & & & & \\
\hline 038 & Visible & & & & \\
\hline 0,56 & Visible & Brun & & & \\
\hline 0,69 & Visible & Brun & & & \\
\hline 0,81 & Visible & & & & \\
\hline 0,94 & & Vert & & & \\
\hline \multicolumn{5}{|c|}{ Décocté épuisé } & \\
\hline 0,06 & Visible & & \multirow{8}{*}{1} & \multirow[t]{8}{*}{ Terpènes } & \multirow{8}{*}{8} \\
\hline 0,23 & Visible & & & & \\
\hline 0,31 & Visible & & & & \\
\hline 0,59 & Visible & & & & \\
\hline 0,71 & Visible & & & & \\
\hline 0,79 & Visible & Vert & & & \\
\hline 0,81 & Visible & & & & \\
\hline 0,94 & Visible & & & & \\
\hline
\end{tabular}


- Eluant : Butanol-Acide acétique-Eau (40-10-50)

L'anisadéhyde a révélé essentiellement des saponosides (tableau VI et figure 4) dans le digesté et le décocté épuisé 3 composés chacun. Ils contiennent aussi d'autres substances ( 7 et 8 respectivement).

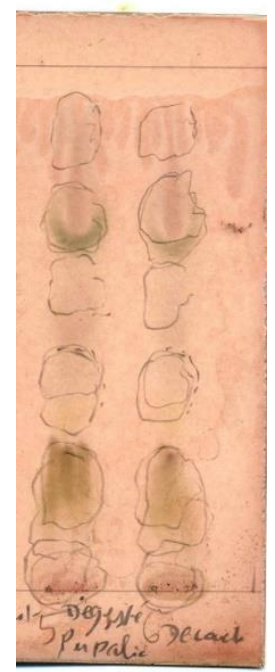

Figure 4 : Saponosides révélés par l'anisaldéhyde dans le visible

Tableau VI : Observation du chromatogramme avant et après révélation par l'anisaldéhyde

\begin{tabular}{|c|c|c|c|c|c|}
\hline $\mathrm{Rf}$ & $254 \mathrm{~nm}$ & Anisaldéhyde & $\begin{array}{c}\text { Nombre } \\
\text { de } \\
\text { composés } \\
\text { identifiés }\end{array}$ & $\begin{array}{l}\text { Groupes } \\
\text { de } \\
\text { composés } \\
\text { identifiés }\end{array}$ & $\begin{array}{l}\text { Nombre otal } \\
\text { de composés }\end{array}$ \\
\hline \multicolumn{4}{|c|}{ Digesté $50^{\circ} \mathrm{C}$} & & \\
\hline 0,06 & Visible & & \multirow{7}{*}{3} & \multirow{7}{*}{$\begin{array}{c}\text { Saponosides } \\
\text { Triterpènes }\end{array}$} & \multirow{7}{*}{7} \\
\hline 0,19 & & Noir & & & \\
\hline 0,21 & Visible & & & & \\
\hline 0,38 & & Noir & & & \\
\hline 0,43 & Visible & & & & \\
\hline 0,65 & & Vert & & & \\
\hline 0,75 & Visible & & & & \\
\hline \multicolumn{4}{|c|}{ Décocté épuisé } & & \\
\hline 0,06 & Visible & & \multirow{8}{*}{3} & \multirow{8}{*}{$\begin{array}{c}\text { Saponosides } \\
\text { Triterpènes }\end{array}$} & \multirow{8}{*}{8} \\
\hline 0,19 & & Noir & & & \\
\hline 0,21 & Visible & & & & \\
\hline 0,39 & & Noir & & & \\
\hline 0,44 & Visible & & & & \\
\hline 0,6 & Visible & & & & \\
\hline 0,63 & & Vert & & & \\
\hline 0,75 & Visible & & & & \\
\hline
\end{tabular}




\section{Pouvoir antioxydant}

- phase mobile : Butanol -Acide acétique - Eau (60-15-25). Le digesté et le décocté épuisé présentent des spots de décoloration du DPPH alors que l'infusé et le décocté n'ont que 1 et 2 tâches respectivement (tableau VII).

Tableau VII : Observation du chromatogramme après révélation par le DPPH

\begin{tabular}{|c|c|c|}
\hline $\mathrm{Rf}$ & DPPH & $\begin{array}{l}\text { Nombre de composés qui } \\
\text { décolorent le DPPH }\end{array}$ \\
\hline \multicolumn{3}{|c|}{ Infusé } \\
\hline 0,4 & Tâche de décoloration & 1 \\
\hline \multicolumn{3}{|c|}{ Décocté } \\
\hline 0,09 & Tâche de décoloration & \multirow[t]{2}{*}{2} \\
\hline 0,4 & Tâche de décoloration & \\
\hline \multicolumn{3}{|c|}{ Digesté } \\
\hline 0,05 & \multirow[t]{8}{*}{ Tâches de décoloration } & \\
\hline 0,08 & & \\
\hline 0,19 & & \\
\hline 0,38 & & \\
\hline 0,56 & & \\
\hline 0,69 & & \\
\hline 0,81 & & \\
\hline 0,94 & & \\
\hline \multicolumn{3}{|c|}{ Décocté épuisé } \\
\hline 0,05 & \multirow[t]{8}{*}{ Tâches de décoloration } & \\
\hline 0,21 & & \\
\hline 0,3 & & \\
\hline 0,41 & & \\
\hline 0,56 & & \\
\hline 0,7 & & \\
\hline 0,84 & & \\
\hline 0,98 & & \\
\hline
\end{tabular}

La Figure 5 (A et B) montre les profils chromatographiques du criblage chimique et ceux de l'activité antiradicalaire. 


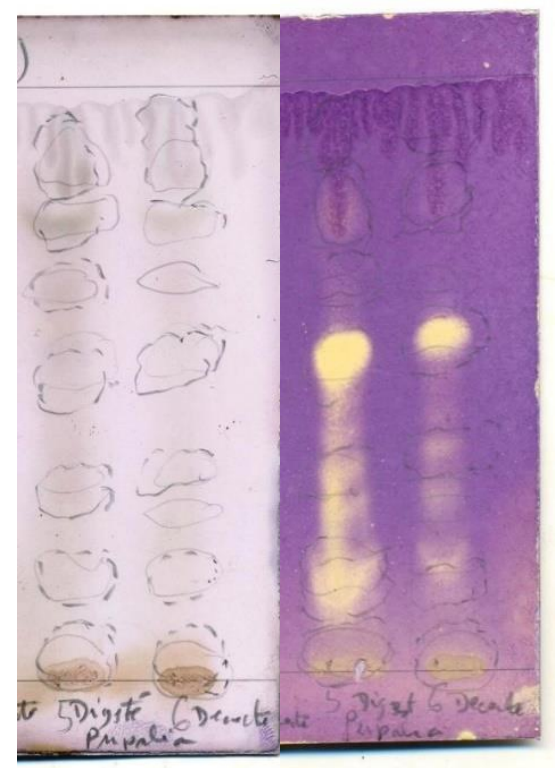

A

B

Figure 5 : Composés terpéniques (A) des extraits aqueux révélés par le réactif de Godin et leurs spots de décoloration du DPPH (B)

- Eluant : Acétate d'éthyle - Méthyléthylcétone - Acide formique - Eau (53-1-1)

Dans ce système de solvants les deux extraits épuisés présentent un spot de décoloration (tableau VIII et figure 6 A et B).

Tableau VIII : Observation du chromatogramme après révélation par le DPPH

\begin{tabular}{|c|c|c|}
\hline \multicolumn{2}{|c|}{ Tâches observées } \\
\cline { 1 - 2 } Rf & $254 \mathrm{~nm}$ & \multirow{2}{*}{ DPPH } \\
\hline \multicolumn{2}{|c|}{ Digesté de décoloration } \\
\hline 0,06 & Visible \\
\hline 0,21 & Visible \\
\hline 0,31 & Visible & \multirow{2}{*}{ Spot de décoloration } \\
\hline 0,5 & Visible \\
\hline 0,71 & Visible \\
\hline 0,88 & Disible \\
\hline \multicolumn{2}{|c|}{ Décocté épuisé } \\
\hline 0,06 & Visible \\
\hline 0,12 & Visible \\
\hline 0,19 & Visible \\
\hline 0,5 & Visible \\
\hline 0,56 & \\
\hline 0,75 & \\
\hline 0,81 &
\end{tabular}




\begin{tabular}{|l|l|l|}
\hline 0,93 & Visible & \\
\hline 0,96 & & \\
\hline
\end{tabular}

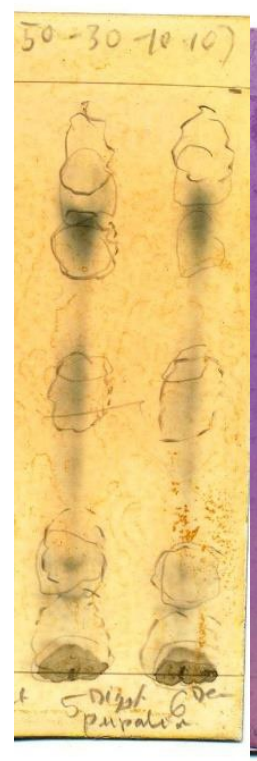

A

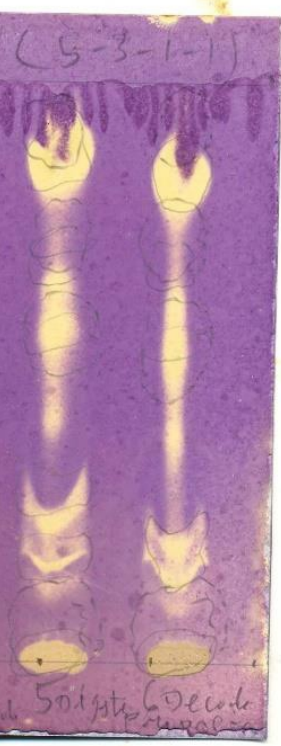

B

Figure 6 : Composés phénoliques (A) des extraits aqueux et leurs spots de décoloration du DPPH (B)

\section{Discussion- Conclusion}

Les groupes de composés dont la présence a été caractérisée par les révélateurs chimiques sont: les saponosides, stérols, terpènes, lactones sesquiterpéniques, tanins et composés phénoliques. Le nombre important de composés des groupes moléculaires identifiés d'abord dans le visible et l'UV à $366 \mathrm{~nm}$ puis après révélation par les différents réactifs montre la richesse en substances chimiques bioactives des extraits. Mais l'extrait méthanolique et les extraits aqueux concentrent plus de composés phénoliques, tanins, que les autres extraits.

La plus forte activité antiradicalaire a été observée avec le digesté et le décocté épuisé après migration dans le système de solvants Acétate d'éthyle Méthyléthylcétone - Acide formique - Eau (5-3-1-1), qui s'est traduit par des bandes de décoloration obtenues après révélation avec le DPPH.

L'activité antioxydante par piégeage de radicaux libres peut être due aux composés phénoliques, aux coumarines ou aux substances terpéniques (Kabran, 2011). La plus importante activité antiradicalaire de notre échantillon serait liée aux composés phénoliques révélés lors du screening chimique par le chlorure de fer le système de solvants Acétate d'éthyle - Méthyléthylcétone - Acide formique - Eau (5-3-1-1). 
Une autre activité antiradicalaire a été décelée sous forme de spots de décoloration du DPPH avec le système de solvants BAW (60-15-25). Il s'agit probablement de composés terpéniques d'après le criblage chimique effectué par le réactif de Godin après migration dans le même éluant.

Les autres extraits aqueux présentent une faible activité antioxydante (infusé : 1 tâche de décoloration ; décocté : 2 tâches de décoloration), après migration dans l'éluant BAW (60-15-25).

Selvan (2014) affirmait que l'extrait méthanolique des parties aériennes de $P$. lappacea a un pouvoir antioxydant sur le DPPH, la Superoxyde dismutase, l'oxyde nitreux, la polyphénol oxydase et la catalase comparées avec l'acide ascorbique comme standard. D'après Naidu et al (2014), des études expérimentales in vitro ont montré que l'extrait éthanolique de $P$. lappacea possède des propriétés antioxydantes et anticancéreuses.

Dans la thérapeutique traditionnelle, $P$. lappacea est utilisée sous forme d'extrait aqueux pour traiter les hémorroïdes (Mamadou et al, 2017 b). Le pouvoir antioxydant révélé sur les extraits aqueux nous permet de déduire que l'usage de la plante étudiée comme antihémorrö̈daire est bien fondé. Par ailleurs, de meilleures propriétés antihémorroïdaires pourraient être obtenues avec le digesté et le décocté épuisé.

Il serait nécessaire d'étudier la phytochimie et l'activité antiradicalaire du décocté, de l'infusé, en utilisant les mêmes systèmes de solvants que précédemment, puis procéder au dosage des divers composés bioatifs supposés responsables de l'effet antihémorroïdaire (en particulier composés phénoliques, terpènes), dans tous les extraits aqueux (infusé, décocté, digesté, décocté épuisé) et sélectionner la meilleure méthode d'extraction afin d'optimiser l'activité antihémorroïdaire de P. lappacea.

\section{References:}

1. Georgievskii V. P., Komissarenko N. F., Dmitrouk S. E. (1990). Substances bioactives des plantes médicinales. Edition "Naouka" Novosibirsk, 336 p. (Traduit du russe).

2. Harborne J.B. (1998). Phytochemical methods: A guide to modern techniques of plant analysis. Springer, Third ed., London, 320p. ISBN10: 0412572702. ISBN-13:978-0412572708.

3. Kabran G. R., Ambeu N. C., Mamyrbékova-Békro J. A., Békro Y.A. (2011). CCM d'extraits sélectifs de 10 plantes utilisées dans le traitement du cancer du sein en Côte d'Ivoire. European Journal of Scientific Research, 63 (4) : 592-603.

4. Ladyguina E. Y. ,Safronitch L. N. , Otriachenkova V. E., Bolandina I. A., Grinkevitch N. I.. (1983). Analyse chimique des plantes médicinales. Edition Moska, Vischaya Chkola : 46-347. 
5. Lagnika L. (2005). Etude phytochimique et activité biologique de substances naturelles isolées de plantes béninoises. Thèse de doctorat, Université Louis Pasteur (Strasbourg/ France), 268 p.

6. Mamadou A. J., Saley K., Boubé M., Rokia S., Saadou M. (2017a). Plantes médicinales et leurs recettes utilisées dans le traitement des hémorroïdes par les tradipraticiens des régions de Niamey et Tillabéri, Niger. Annales de l'Université Abdou Moumouni de Niamey (sous presse).

7. Mamadou A. J., Saley K., Boubé M., Rokia S., Saadou M. (2017b). Etude pharmacognosique de Pupalia lappacea (L.) Juss. Amaranthaceae. Annales de l'Université Abdou Moumouni de Niamey (sous presse).

8. Mamyrbékova-Békro J. A., Konan M. K., Békro Y. A., Djié Bi M. G., Zomi Bi T. J., Mambo V., Boua B. B. (2008). Phytocompound of the extracts of four medicinal plants of Côte d'Ivoire and assessment of their potential antioxidant by thin layer chromatography. European Journal of Scientific Research, 24 (2): 219-228.

9. Merck E. (1980). Révélateurs pour la chromatographie en couche mince et sur papier. Darmstadt : 12-153.

10. Naidu P. P., Madakka M., Rajesh B. (2014). Pupalia lappacea juss [L]: a review of phytochemistry and therapeutic application. Asian Journal of Pharmaceutical and Clinical Research, 7(Suppl. 1): 15-18.

11. OUA/CSTR. (1988). Pharmacopée africaine, méthodes générales d'analyse. Edn 1 Publisher, Lagos (Nigeria), 1988, 254p.

12. Selvan A. T., Subramanian N. S., Ramadevi M., Prasad S. G., Muthu S. K. Bioactive compound identification, phytochemical estimation, in-vitro anti-inflammatory and antioxidant activity of Pupalia lappacea. International Journal of Pharmacognosy Panchkula, India, 2014, 1(9): 596-604.

13. Sofowora A. (1993). Screening Plants for Bioactive Agents In: Medicinal Plants and Traditional Medicine in Africa. John Wiley \& Sons Ltd, 274 p. ISBN-13: 978-0471103677.

14. Takao T., kitatami F., Watanabe N., Yagi A. and Sakata K. (1994). A simple screening method for antioxidants and isolation of several antioxydants produced by marine bacteria from fish and shell fish. Bioscience, Biotechnologie and biochemistry, 58: 1780-1783.

15. Wagner H., Bladt S., Zgainski E. (1996). Plant drug analysis, a thinlayer, chromatography atlas. Springer Verlag, Berlin Heidlberg, $2^{\text {nd }}$ ed. New York, 320 p. 